\title{
Remote colorimetric measurements by hyperspectral lidar compared to contact conventional colorimetry
}

\author{
Federico Angelini $^{1}$ | Anna C. Felici ${ }^{2}$ | Martina Franchi $^{3}$ | Valeria Spizzichino $^{1}$ ()
}

${ }^{1}$ ENEA - Research Center of Frascati, Frascati, Italy

${ }^{2}$ Department of Basic and Applied Science for Engineering, University of Rome "La Sapienza", Rome, Italy

${ }^{3}$ ENEA, Frascati, Italy

\section{Correspondence}

Valeria Spizzichino, ENEA - Research Center of Frascati. Via E.Fermi 45 00044, Frascati (Rome), Italy.

Email: valeria.spizzichino@enea.it

Funding information

Regione Lazio

\begin{abstract}
Lidars have many applications in different fields, including the field of Cultural Heritage. The purpose of this study is to evaluate a scanning hyperspectral lidar prototype developed at ENEA Center of Frascati as a useful tool for colorimetric analysis when samples cannot be studied with standard contact instruments. A certified X-Rite Color-checker sample, consisting of 24 colored blocks with a coordinated colorimetric certificate, was analyzed. In order to obtain colorimetric data from the reflectance spectra, the precepts of the Commission de l'Éclairage were followed and an algorithm was developed to calculate the color difference between the certified values of the Color-checker and commercial and non-commercial spectrophotometers and those obtained by the lidar, confirming this latter as a useful tool for remote colorimetry.
\end{abstract}

\section{K E Y W O R D S}

colorimetry, $L^{*} a^{*} b^{*}$, lidar, reflectance

\section{1 | INTRODUCTION}

Quantitative measurement of light may be considered under several points of view. On one hand, colorimetry represents the measurement of color considering the multiple processes that participate in the visual experience; on the other hand, spectrophotometry only refers to quantitative measurements of light reflection and transmission of light by materials. Colorimetry takes into account the processes that involve light, colored objects and observer ${ }^{1}$ and plays a role of primary importance in the monitoring of Cultural Heritage. ${ }^{2}$ Artworks are subjected to the incessant passing of time; hence colorimetry is a useful tool to understand how much and in which way the original colors change. ${ }^{3}$ Under this perspective, in fact, colorimetric measurements provide more significance than spectrophotometric data, because the color immediately characterizes a surface being detectable with the naked eye. Spectrophotometers retrieve instead the amount of light reflected by a sample at many wavelengths, usually in the visible (VIS) region (380-740 nm).
If the signal is collected in separate bands, the collection method is called multispectral, if the bands are close together (regardless the width of each band) so that the sampling completely fills the wavelength range, the collection method is called hyperspectral. The obtained vector represents the reflectance spectrum, a very significant characteristic of a target since it influences the colors perceived by human eye, through the interaction with the three kinds of retinal cones that determine the red, green and blue components in color. The $\bar{x}, \bar{y}, \bar{z}$ functions, referred to as tristimulus matching functions, were determined in the "20s" by the International Commission de l'Éclairage (CIE) to reproduce the cone spectral response to light at different wavelengths. These functions were measured on human observers for fields of view of $2^{\circ}$ and $10^{\circ}$, depending on the application and the ensemble of these three functions are referred to as the CIE standard observer. The convolution of the tristimulus weighting functions with the radiance spectrum give a threedimensional vector, the XYZ tristimulus triplet. This allows to describe color sensations by a triplet of numbers 
instead of a complete spectrum. While in tristimulus colorimeters the convolution is made by using calibrated color filters that reproduce the tristimulus matching functions, in spectrophotometers the convolution is numerically performed.

There are several instruments used in the colorimetric measurements-from spectrophotometers to colorimeters-and new instruments are being developed. They are based on the measurement of the chromatic aspects of the light reflected by surfaces illuminated by a source, placing the instrument in contact with the sample. These instruments can be also transportable, allowing measurements to be made in situ. Such instruments are generally equipped with software operating according to algorithms unknown to the operator, therefore it is difficult to understand how the obtained data are processed and how to compare them to data coming from a different colorimeter. The only way to assess the quality of an instrument and its software globally is to take measurements on color samples certified by a metrological laboratory and to evaluate the difference between measured and certified values. ${ }^{4}$ These instruments, because of their structure and operating principles, encounter several difficulties on samples with large and inhomogeneous surfaces. Artworks, instead, often present irregular and/or curved surfaces difficult to be measured by a rigid contact instrument, which requires full adhesion to the surface to have a full control on the geometry of illumination and collection of reflected light. Remote (up to tens of meters) color measurements would be very attractive when instrumental adhesion to the sample is compromised, or when it presents so delicate surfaces that any contact has to be avoided.

In this study a lidar scanning prototype system developed at FSN-TECFIS-DIM Laboratory of ENEA Research Center of Frascati has been tested as a remote tool for colorimetry. In fact, the lidar allows to study the reflectance spectra of the samples by performing remote measurements, even in situ, and to record large quantity data faster with respect to commercial punctual colorimeters.

For this study normative procedures given by the CIE were followed. ${ }^{5-8}$ The CIELAB $1976 \mathrm{~L}^{*} \mathrm{a} * \mathrm{~b}^{*}$ color space, and the $L^{*} a^{*} b^{*}$ color coordinates were introduced with the specific aim to obtain a direct correspondence between the amount of numerical change in coordinates and the amount of visually perceived change, so the most natural way to compare results is through the color difference $\Delta E_{\mathrm{ab}}^{*}{ }^{*}$ When introduced, all three coordinates contributed equally to the total distance; this concept has been refined many times since then taking into account deviations from perceptual uniformity. However, a thorough analysis of color differences is beyond the aims of this work, and the original $\Delta E_{\mathrm{ab}}^{*}$ will be identified as $\Delta E$ has been adopted hereafter in this article. Moreover, the
$L^{*} a^{*} b^{*}$ coordinates are completely device-independent, so they represent the best way to compare color measurements from different instruments.

Lidar reflectance measurements were carried out with $45^{\circ}: 0^{\circ}$ measurement geometry and the CIE standards were followed to process the reflectance signal to derive the colorimetric data. To test the efficiency of the system and the software developed, a reference sample consisting of an X-Rite color-checker was used. ${ }^{10}$ The results of the lidar were compared to those obtained using two different commercial spectrophotometers (the CM-2600d Konica Minolta and the ColorTest Elektrophysik), and a fiber optics reflectance spectroscopy system (FORS). Hence, data coming from each one of the aforementioned instruments were compared to the colorimetric values provided by X-Rite and the $\Delta E$ were calculated. Finally, the variability of the instrumental values was assessed to define the reliability of the measurements with the lidar prototype system.

\section{2 | MATERIALS AND METHODS}

\section{1 | Reference samples}

An X-Rite color-checker Classic was used as reference. This is a certified sample used for digital camera calibration, composed of 24 colored blocks $(5 \mathrm{~cm} \times 5 \mathrm{~cm})$ which represent natural colors-similar to foliage and human skin- and their shade for different degrees of saturation.

Primary colors RGB-CMY, white and scale of grays are represented as well (https://www.xrite.com/categories/ calibration-profiling/colorchecker-classic, s.d.). For each block colorimetric coordinates are given into color space sRGB and CIE $L^{*} a^{*} b^{*}$ by the manufacturer. CIE $L^{*} a^{*} b^{*}$ coordinates are given for a $2^{\circ}$ observer and a D50 illuminant, while sRGB coordinates for a $2^{\circ}$ observer and a D65 illuminant, in a measurement geometry $45^{\circ}: 0^{\circ}$. A representation of the color-checker is given in Figure 1. The colors and the relative colorimetric coordinates are listed in Table 1. The values in the table are the data provided by the manufacturer that specifies that they are an average of values for all the produced charts, therefore the single sample can deviate slightly from the value reported. The manufacturer does not provide any $\Delta E$ that could quantify how much each color can deviate from the reported value. Moreover, in any chart, fluctuations can be found point by point for each color. Although such fluctuations (measured as SD of $\Delta E$ among different points in the same square) result to be smaller than the just noticeable difference (JND) accepted in the field of color perception, non-uniformities for some colors are large enough to be detected by the high-end colorimeters and spectrophotometers employed in this work. 


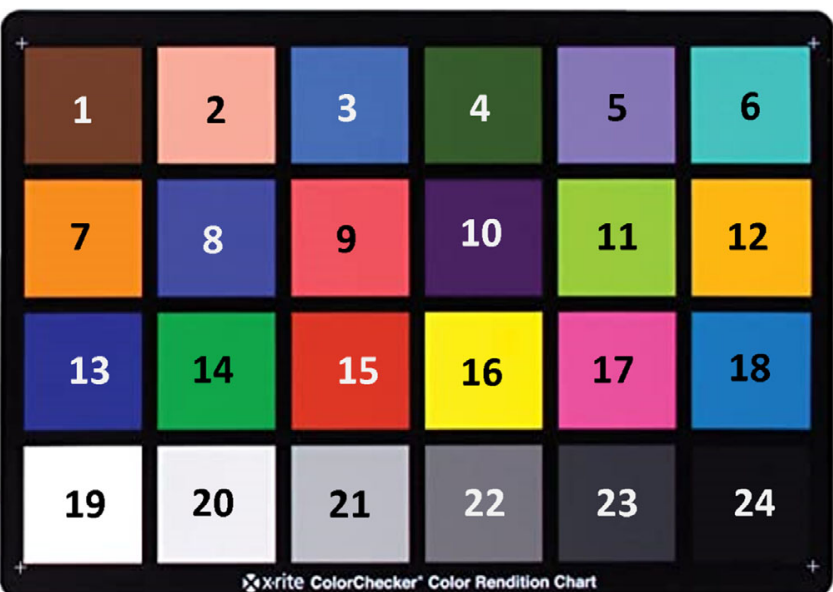

F I G U RE 1 The color-checker used as reference for algorithm validation. Superimposed numbers correspond to the colors described in Table 1
In order to calibrate the measurements from the lidar prototype scanning system, two additional targets, one target white and one black, were inserted into the scene. These targets are coated by thick layers of white acrylic paint Chrèon Framatix white pro, and Saratoga Happy color, black matte RAL 9005, respectively. These paints were selected as the most and least reflective (in terms of integrated reflectance) after several laboratory tests. They were used as references for the white spectrum and the dark signal for determining the reflectance spectra of all other points. More details on this procedure will be given in in the following.

\subsection{Instrumentation}

As explained above, reflectance and colorimetric data coming from a prototypal lidar system were compared
T A B L E 1 Colors list pertaining to the X Rite color-checker Classic

\begin{tabular}{|c|c|c|c|c|c|c|c|}
\hline \multirow[b]{2}{*}{ Color number } & \multirow[b]{2}{*}{ Name } & \multicolumn{3}{|c|}{ sRGB } & \multicolumn{3}{|c|}{ CIE $L^{*} \mathbf{a}^{*} \mathbf{b}^{*}$} \\
\hline & & $\boldsymbol{R}$ & $G$ & $\boldsymbol{B}$ & $L^{*}$ & $a^{*}$ & $b^{*}$ \\
\hline 1 & Dark skin & 115 & 82 & 68 & 37.986 & 13.555 & 14.059 \\
\hline 2 & Light skin & 194 & 150 & 130 & 65.711 & 18.130 & 17.810 \\
\hline 3 & Blue sky & 98 & 122 & 157 & 49.927 & -4.880 & -21.925 \\
\hline 4 & Foliage & 87 & 108 & 67 & 43.139 & -13.095 & 21.905 \\
\hline 5 & Blue flower & 133 & 128 & 177 & 55.112 & 8.844 & -25.399 \\
\hline 6 & Bluish green & 103 & 189 & 170 & 70.719 & -33.397 & -0.199 \\
\hline 7 & Orange & 214 & 126 & 44 & 62.661 & 36.067 & 57.096 \\
\hline 8 & Purplish blue & 80 & 91 & 166 & 40.020 & 10.410 & -45.964 \\
\hline 9 & Moderate red & 193 & 90 & 99 & 51.124 & 48.239 & 16.248 \\
\hline 10 & Purple & 94 & 60 & 108 & 30.325 & 22.976 & -21.587 \\
\hline 11 & Yellow green & 157 & 188 & 64 & 72.532 & -23.709 & 57.255 \\
\hline 12 & Orange yellow & 224 & 163 & 46 & 71.941 & 19.363 & 67.857 \\
\hline 13 & Blue & 56 & 61 & 150 & 28.778 & 14.179 & -50.297 \\
\hline 14 & Green & 70 & 148 & 73 & 55.261 & -38.342 & 31.370 \\
\hline 15 & Red & 175 & 54 & 60 & 42.101 & 53.378 & 28.190 \\
\hline 16 & Yellow & 231 & 199 & 31 & 81.733 & 4.039 & 79.819 \\
\hline 17 & Magenta & 187 & 86 & 149 & 51.935 & 49.986 & -14.574 \\
\hline 18 & Cyan & 8 & 133 & 161 & 51.038 & -28.631 & -28.638 \\
\hline 19 & White & 243 & 243 & 242 & 96.539 & -0.425 & 1.186 \\
\hline 20 & Neutral 8 & 200 & 200 & 200 & 81.257 & -0.638 & -0.335 \\
\hline 21 & Neutral 6.5 & 160 & 160 & 160 & 66.766 & -0.734 & -0.504 \\
\hline 22 & Neutral 5 & 122 & 122 & 121 & 50.867 & -0.153 & -0.270 \\
\hline 23 & Neutral 3.5 & 85 & 85 & 85 & 35.656 & -0.421 & -1.231 \\
\hline 24 & Dark & 52 & 52 & 52 & 20.461 & -0.079 & 0.973 \\
\hline
\end{tabular}

Note: CIE $L^{*} a^{*} b^{*}$ coordinates are given for a $2^{\circ}$ observer and a D50 illuminant, while sRGB coordinates for a $2^{\circ}$ observer and a D65 illuminant, in a measurement geometry $45^{\circ}: 0^{\circ}$. 
with those obtained using 2 different commercial contact spectrophotometers, ColorTest Elektrophysik and CM2600d Konica Minolta and a system for FORS. These instruments were selected in order to compare systems based on similar technology but with different proprietary software (Minolta and Elektrophysik) and systems based on the use of different layouts and components (FORS).

\subsection{1 | Commercial instruments for colorimetric measurements}

\section{Elektrophysik ColorTest}

ColorTest by Elektrophysik is a portable spectrophotometer equipped with a flexible measuring probe, with a circular illumination at $45^{\circ}$ through LED lights and a $45^{\circ} \mathrm{a}: 0^{\circ}$ measurement geometry in accordance to DIN-Norm 5033. The instrument is calibrated by a BAM certified white standard provided by the spectrophotometer manufacturer. The standard illuminants D65, D50, A, C, as well as $2^{\circ}$ or $10^{\circ}$ observer can be selected. The instrument operates in the spectral range between 400 and $700 \mathrm{~nm}$ with $10 \mathrm{~nm}$ resolution; the sampled area diameter is $8 \mathrm{~mm}$ large. From the reflectance spectrum the spectrophotometer instantly gives the colorimetric coordinates in different color spaces through a dedicated software provided by the manufacturer. The system uses the software ColorDaTra. No details about the algorithms used are provided.

\section{CM-2600d Konica Minolta}

A CM-2600d from Konica Minolta commercial portable spectrophotometer was also used. This instrument, compliant with CIE No. 15, ASTM E1164, DIN 5033, is equipped with a diffuse illumination, an $8^{\circ}$ viewing and can operate in both SCI (Specular Component Included) and SCE (Specular Component Excluded) configurations. For this study, the SCE configuration and a sampling area diameter of $8 \mathrm{~mm}$ were considered. The standard illuminants provided by the instrument are A, C, D50, D65, F2, F6, F7, F8, F10, F11, F12. The instrument operates between 360 and $740 \mathrm{~nm}$ with $10 \mathrm{~nm}$ resolution. The system uses the software SpectraMagic NX.

\section{Fiber optics reflectance spectroscopy}

Fiber optics reflectance spectroscopy was performed with an Avantes AvaSpec spectrophotometer equipped with a CCD linear sensor (2048 pixels) and a diffraction grating with 300 lines $/ \mathrm{mm}$, blazed at $500 \mathrm{~nm}$, which covers a spectral range from 300 to $1100 \mathrm{~nm}$ with a spectral resolution of $0.8 \mathrm{~nm}$. The illuminator was a halogen lamp (HL-2000 FHSA, Avantes). The measurement geometry was $2 \times 45^{\circ}: 0^{\circ}$, through a bifurcated fiber (diameter of
$600 \mu \mathrm{m})$ for the illumination and a $200 \mu \mathrm{m}$ diameter fiber (NA $0.22 \pm 0.02$ ) for the reflected light. The sampled area diameter is $1 \mathrm{~mm}$. The system uses the Avantes software AvaSoft-COL.

\subsection{2 | Lidar system}

The scanning lidar apparatus has been developed at the Diagnostic and Metrology Laboratory of the ENEA Frascati research center for remote fluorescence measurements. Usually this system uses as light source a compact pulsed, diode pumped, solid state laser, emitting UV radiation at $266 \mathrm{~nm}$ suitable for the fluorescence excitation of the targets under study. A set of optics (mirrors, lens and quartz fiber optic) allows to transmit the exciting laser radiation and to receive the scattering and fluorescence signals from the investigated target. The collecting mirror is actuated by two high accuracy rotating stepping motors allowing for a scan of a selected surface in order to record from every single point of the target a whole spectrum. The backscattered radiation is optically driven to a collecting lens and focused at the entrance of a fiber optic (SMA 905 single-strand optical fiber 0.22NA), linked to a compact QE-Pro spectrometer (Ocean Optics). The cooled CCD detector (Hamamatsu S7031-1006 scientific grade, back-thinned) in the spectrometer can record the broad spectral emission with $2.5 \mathrm{~nm}$ resolution in the range from 200 up to $900 \mathrm{~nm}$, resulting in hyperspectral sampling. The working distance spans from $50 \mathrm{~cm}$ to some meters, with a spatial resolution of about $2.5 \mathrm{mrad}(2.5 \mathrm{~mm}$ at $1 \mathrm{~m}$ distance). The scheme of the prototype if reported in Figure 2.

The same system can be used for reflectance measurements, using wide-spectrum lamps installed with appropriate geometrical layout instead of the laser. Thanks to this ability, the system has often been used to obtain superimposable maps of fluorescence and reflectance. In fact, the complementarity between these data provides a more complete surface characterization and interesting information on the composition of the target. ${ }^{11,12}$ It is clear, therefore, how the transition from reflectance to colorimetric coordinates would constitute a further step forward for the interpretation and usability of the data.

Then, for the present work, two halogen R7s lamps were used. They can be assumed as standard illuminant $\mathrm{A}$, which shows a correlated color temperature of about $2900^{\circ} \mathrm{C}$. Anyway, the exact properties of the light source are not of particular importance, since normalization on a white target is performed before calculating the reflectance spectra. The crux is whether sufficient radiance is irradiated in each part of the collected spectrum, in order to avoid noisy data or blind bands in dark spectral regions of the light source. The spectral 
F I G U RE 2 Scheme of the lidar system used and its measurement geometry

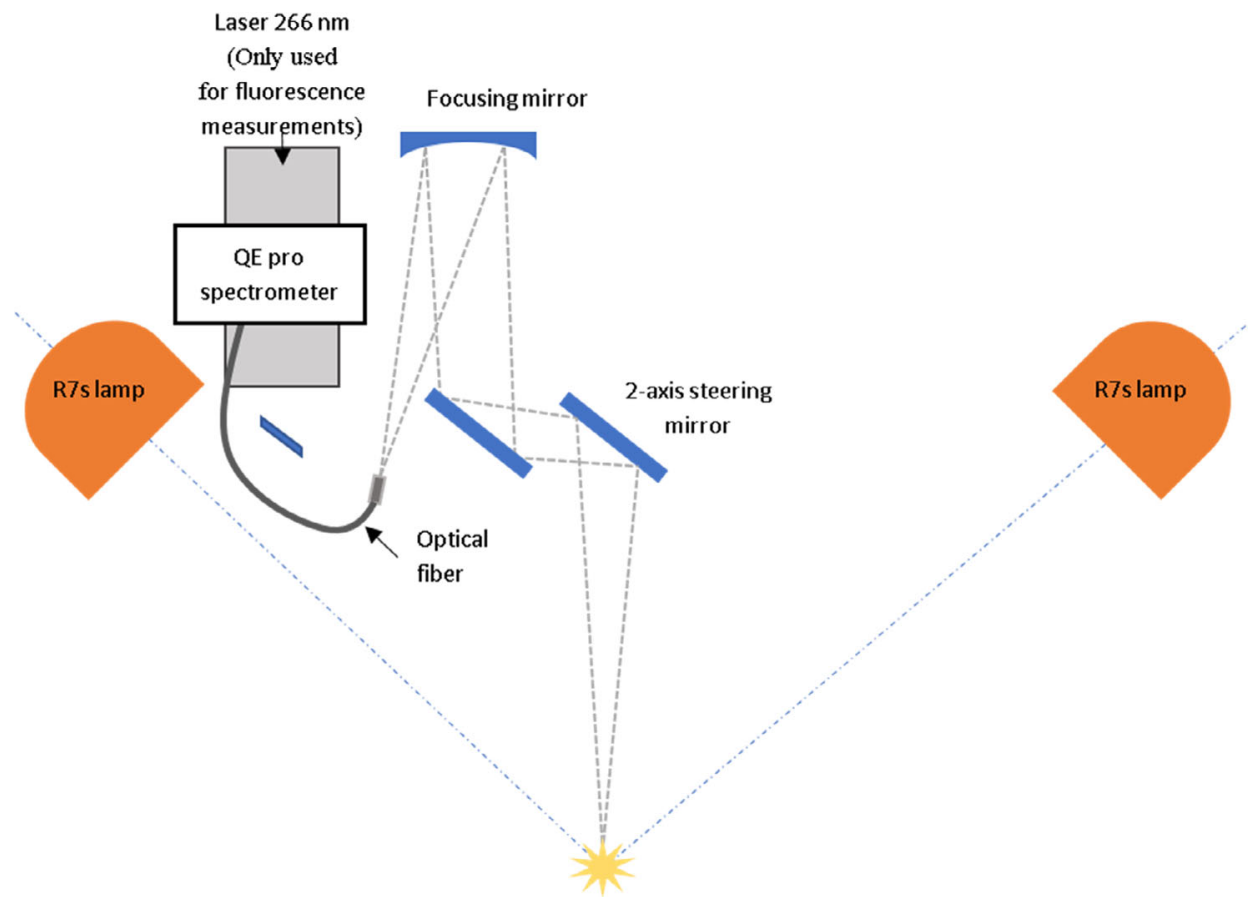

radiance $\mathrm{f}$ R7s lamps at $400 \mathrm{~nm}$ is about $8 \%$ of the one at $700 \mathrm{~nm}$.

To compare the results coming from the presented instruments properly, the coherence among experimental conditions is fundamental. Moreover, the choice of lighting geometry and illuminant, which must comply with CIE regulations, ${ }^{7,13}$ is of primary importance. In fact, different configurations in measurement geometry lead to different results $^{14}$; therefore, when possible, approximately the same geometries $45^{\circ}: 0^{\circ}$ for illumination and collection were chosen. For the CM-2600d Konica Minolta spectrophotometer this was not possible, because it is equipped with an integrating sphere and, as described in section "CM-2600d Konica Minolta," the lighting system is diffused and the signal is collected at $8^{\circ}$. Therefore, only the SCE measurements were taken into account, as they are more comparable with the measurements obtained with the instruments with a $45^{\circ}: 0^{\circ}$ geometry. The illuminating geometry for lidar was set by illuminating the samples at $45^{\circ}$ with respect to the normal by two halogen lamps, collecting signal scattered at $0^{\circ}$ (ie, normally), without any contact with the sample (measurement geometry $2 \times 45^{\circ}: 0^{\circ}$ ).

For the colorimetric analysis, the $2^{\circ}$ observer was set for all the instruments; the CIE $L^{*} a^{*} b^{*}$ and SRGB color spaces were chosen to show the colorimetric data.

As for the CIE $L^{*} a^{*} b^{*}$ coordinates, a D50 illuminant was chosen, while D65 illuminant was chosen for sRGB coordinates. A scheme of the conditions set for the four instruments used is reported in Table 2.

Once the most appropriate measurement conditions for each instrument was selected, a suitable software for the processing of lidar data, based on algorithms in agreement with the CIE regulations, have been developed. Methods implemented in such software are presented in the next section.

\section{3 | Algorithms}

To calculate the colorimetric data from the reflected fraction of light by the surface of the sample, the following steps are necessary ${ }^{4}$ :

1. Measurement of the reflectance factor $R(\lambda)$ according to defined geometry (CIE Publication N.176:2006, 2006)

2. Conversion to colorimetric coordinates in relation to the illuminant and observer considered.

As far as lidar measurements are concerned, the signal acquired by the spectrometer is given in arbitrary units. A white target allows the white point calibration: this means that, considering its reflectance as identically unitary, its spectrum can be used as a normalization function to eliminate the contribution of the illuminant on the detected signal, at least if enough radiance is available at each wavelength.

On the other hand, also ambient straylight (more and more important as the target-to-detector distance increases) and dark signal affect the sensor, giving a nonzero signal also in the darkest point of the image. This leads to the impossibility to acquire a true black color, and to do that a dark subtraction should be performed. A 
T A B L E 2 Scheme of conditions set for color space sRGB (top) and CIELAB (bottom)

\begin{tabular}{llllll} 
Instrument & $\begin{array}{l}\text { Illuminant } \\
\text { sRGB }\end{array}$ & $\begin{array}{l}\text { Illuminant } \\
\text { CIELAB }\end{array}$ & $\begin{array}{l}\text { Sampling spot } \\
\text { diameter }\end{array}$ & $\begin{array}{l}\text { Measurement } \\
\text { geometry }\end{array}$ & $\begin{array}{l}\text { Standard } \\
\text { observer }\end{array}$ \\
\hline ColorTest & D65 & D50 & $8 \mathrm{~mm}$ & $45^{\circ} \mathrm{a}: 0^{\circ}$ & $2^{\circ}$ \\
CM-2600d & D65 & D50 & $8 \mathrm{~mm}$ & $2 \times 45^{\circ}: 0^{\circ}$ & $2^{\circ}$ \\
FORS & D65 & D50 & $1 \mathrm{~mm}$ & $2 \times 45^{\circ}: 0^{\circ}$ & $2^{\circ}$ \\
lidar & D65 & D50 & $2.5 \mathrm{~mm}$ & $2^{\circ}$ & \\
\hline
\end{tabular}

Abbreviation: FORS, fiber optics reflectance spectroscopy system.

dark-field subtraction can be obtained either through the use of a black matte target within the scene or by acquiring a dark signal, that is, with no illumination of the spectrometer. We decided to include a black target in the scene, to perform an absolute mapping to the range $(0,1)$ for each pixel: this also excludes the contribution of ambient background light, being the reflection of even black targets never null. This whole procedure allows to rescale the signal in a given range, and also acts as a white balancing, making the measurements independent on the light source: as a result, the reflectance spectra are stored and converted into colorimetric coordinates.

Hence, the signal $S(x, y, \lambda)$ from any pixel must include the subtraction of the spectrum of the black point $B=\left(x_{\mathrm{b}}, y_{\mathrm{b}}\right), S_{\mathrm{b}}(\lambda)=S_{0}\left(x_{\mathrm{b}}, y_{\mathrm{b}}, \lambda\right)$ from the raw signal $S_{0}(x, y, \lambda)$ :

$$
S(x, y, \lambda)=S_{0}(x, y, \lambda)-S_{\mathrm{b}}(\lambda)
$$

For an opaque object, at any pixel $x, y$ the target reflectance $R(x, y, \lambda)$ can be defined by the ratio between the spectral radiance $S(x, y, \lambda)$ reaching the observer and the radiance $S_{\text {ill }}(\lambda)$ received by the illuminant:

$$
S(x, y, \lambda)=S_{\mathrm{ill}}(x, y, \lambda) \cdot R(x, y, \lambda)
$$

A certified white target may be employed to exclude the contribution of the illuminant from $S(\lambda)$ and determine the reflectance spectrum of the sample. If the white target may be assumed to equally reflect all the wavelengths in the visible range, the spectrum of the illuminant can be easily derived:

$$
S_{\mathrm{ill}}(x, y, \lambda)=k\left(S_{\mathrm{w}}(x, y, \lambda)-S_{\mathrm{b}}(\lambda)\right)
$$

where $S_{\mathrm{w}}(x, y, \lambda)=S_{0}\left(x_{\mathrm{w}}, y_{\mathrm{w}}, \lambda\right)$ defines the spectrum of the white point $W=\left(x_{\mathrm{w}}, y_{\mathrm{w}}\right)$, which is proportional to the illuminant spectrum. Moreover, under the hypothesis that for reference targets $k \approx 1$, this term can be omitted in practical applications. After the black- and white-point corrections, the reflectance spectrum can be calculated for all the other pixels in the scene simply by inverting Equation (1):

$$
R(x, y, \lambda)=\frac{S_{0}(x, y, \lambda)-S_{\mathrm{b}}(\lambda)}{S_{\mathrm{w}}(\lambda)-S_{\mathrm{b}}(\lambda)}
$$

If we adopt the notation $T=\left(\overline{t_{1}}, \overline{t_{2}}, \overline{t_{3}}\right)=(\bar{x}, \bar{y}, \bar{z})$, the operation may be condensed in one equation:

$$
T_{n}=N \int_{\lambda \min }^{\lambda \max } I(\lambda) R(\lambda) \overline{t_{n}}(\lambda) d \lambda
$$

where $I(\lambda)$ represents the illuminant spectral power distribution, and the normalization factor is given by:

$$
N=1 / \int_{\lambda \min }^{\lambda \max } I(\lambda) \bar{t}_{2}(\lambda) d \lambda
$$

In real case of discrete spectra, we must use the summation:

$$
T_{n}=N \sum_{k} I\left(\lambda_{k}\right) R\left(\lambda_{k}\right) \bar{t}_{n}\left(\lambda_{k}\right) \Delta \lambda_{k}
$$

With:

$$
N=1 / \sum_{k} I\left(\lambda_{k}\right) \overline{t_{2}}\left(\lambda_{k}\right) \Delta \lambda_{k}
$$

Colors of opaque samples naturally depend on the illuminant, in fact color balance in digital imaging represents a very important task for color rendition and automated white balance is still an open issue, as witnessed by the large number of articles and patents on this topic. ${ }^{15} \mathrm{Neg}$ lecting the illuminant in Equation (6) one obtains XYZ coordinates referred to an equal energy illuminant ( $E$ illuminant). If it is requested to simulate color coordinates under a different illuminant, two ways are possible.

First, the theoretical spectrum of the illuminant can be considered in Equation (4) or (6), and the correct tristimulus values will output directly. As an alternative, a series of white balancing transformation matrices on the RGB coordinates have been proposed so far; in this case, 


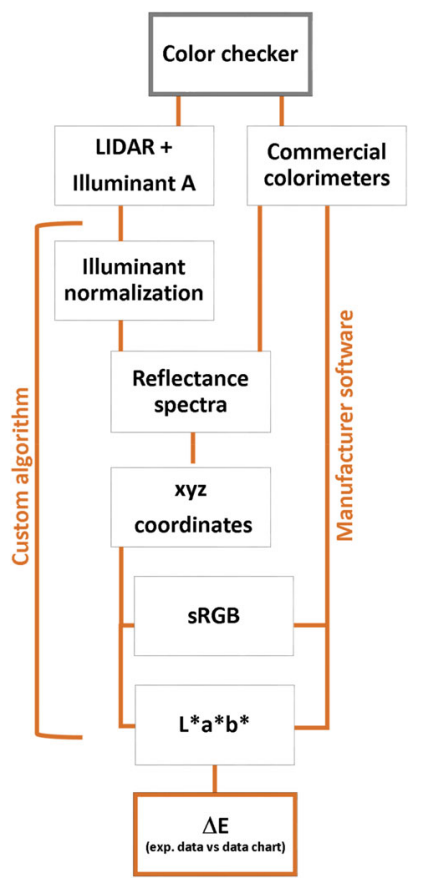

FI G URE 3 The logical scheme of the work here presented

it is possible to get color triples obtained under illuminant $E$ and then balancing the color using the threedimensional transformations. This leads to approximated values, but calculations are easier to perform and compare to RGB sensors.

From XYZ coordinates (CIE 1931), conversion to sRGB and $L^{*} a^{*} b^{*}$ is performed using Matlab functions. In order to obtain sRGB coordinates, the command "xyztorgb" has been used; in fact, for this command Matlab uses the sRGB color space and converts CIE 1931 $\mathrm{XYZ}$ values ( $2^{\circ}$ observer) to sRGB values. For this color space the illuminant D65 is considered by default. In order to get the CIE $1976 L^{*} a^{*} b^{*}$ color values from XYZ (CIE 1931) values set with $2^{\circ}$ observer, the command "xyz2lab" was used, specifying D50 as reference for the white point. We compared the sRGB and $L^{*} a^{*} b^{*}$ coordinates obtained from XYZ using the Matlab function with sRBG and $L^{*} a^{*} b^{*}$ values obtained from XYZ using the functions suggested by CIE (CIE 1976) and IEC 61966-2-1; perfect matching is found.

In Figure 3 a logical scheme of the whole procedure is presented. The results obtained by the four instruments on the reference color-checker will be presented in the next section.

\section{3 | RESULTS}

As illustrated above, here data coming from the four instruments presented have been considered. In

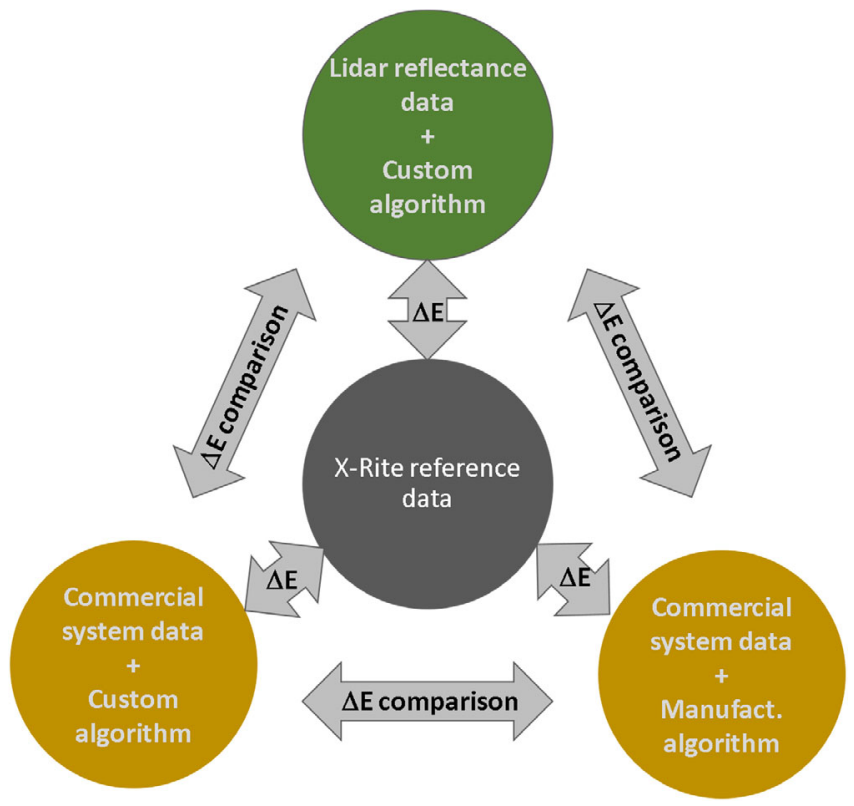

F I G URE 4 Scheme of the comparison carried out by calculation of $\Delta E$ for different colors of color-checker

particular, raw data obtained with the commercial systems have been compared after elaboration by the manufactures software and after processing by the developed custom algorithm. Every experimental data set has been compared to the X-Rite tabulated values provided for the color-checker. A scheme of the value comparisons carried out is presented in Figure 4.

For the three contact instruments, five measurements for each color were carried out and averaged.

For the lidar, a set of nine adjacent pixels were selected and averaged, to reduce the random errors and estimate their consistency. Since in the adopted setup the target is set at $1 \mathrm{~m}$ from the instrument, each pixel is about $2.5 \mathrm{~mm}$ large, and the averaged region covers about $7.5 \times 7.5 \mathrm{~mm}^{2}$. Results showed a very good consistency among lidar spectra, being the relative fluctuations about $3 \%$ at $390 \mathrm{~nm}$, and under $1 \%$ over $450 \mathrm{~nm}$. The larger relative fluctuations in the UV is mainly due to the weaker lamp emission.

From the color values the $\Delta E^{*}{ }_{\mathrm{ab}}{ }^{9}$ was calculated considering the $L^{*} a^{*} b^{*}$ coordinates:

$$
\Delta E_{a, b}^{*}=\left[\left(L_{1}^{*}-L_{2}^{*}\right)^{2}+\left(a_{1}^{*}-a_{2}^{*}\right)^{2}+\left(b_{1}^{*}-b_{2}^{*}\right)^{2}\right]^{1 / 2}
$$

The data highlighted the high reproducibility of the measurements, giving SD values within the JND. Results showed that, for any instrument and for any color, the relative fluctuations (SD/mean value) among repeated measurements are always below $2 \%$. The larger relative fluctuations can be found at both edges of the spectra, 

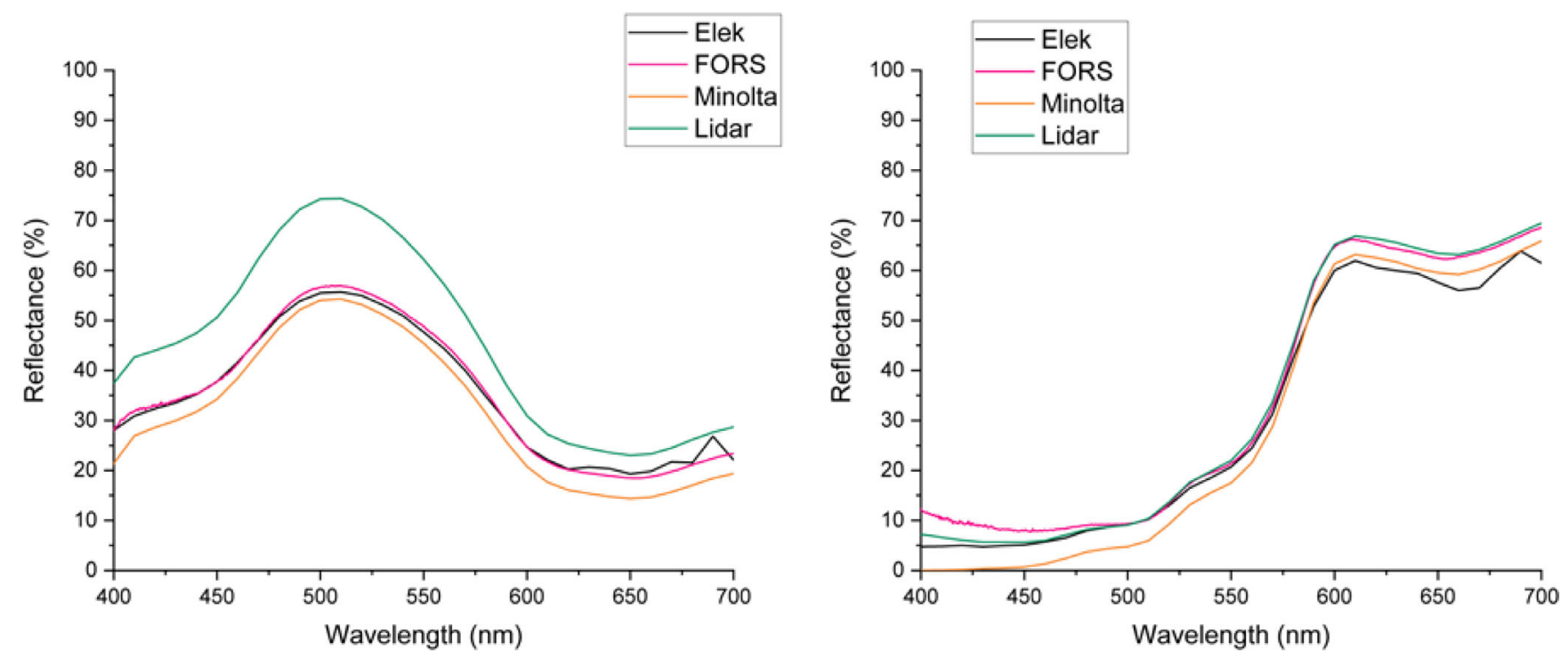

F I G U RE 5 Raw reflectance spectra for colors 6 (bluish green, left) and 7 (orange, right) obtained with the four instruments tested

likely due to either the weaker lamp emission or to a general lower sensitivity of the spectrometers in the lateral regions of the operative spectral range; in the central regions the relative fluctuations are below $1 \%$.

Before comparing reflectance and colorimetric data from the different instruments used for the present work, it is necessary to underline that reproducibility tests performed on different points of every color by the CM2600d have shown that colors of the color-checker are not always homogeneous point by point. The worst result has been obtained for the color 15, red: in this case there is a $\Delta E \mathrm{SD}$ equal to 1.7. To lower the impact of such fluctuations, measurements have been collected on different points of every colors and then averaged. In particular, for the lidar, a set of nine adjacent pixels were selected and averaged, to reduce the random errors and estimate their consistency. For the other instruments five different regions have been selected for each color.

In Figure 5, two examples are reported to highlight different behavior occurring on different colors. While in the spectra on the left (color 6, bluish green) the lidar seems to over-estimate the reflectance in comparison to the other instruments, the spectra on the right (color 7 , orange) show a good agreement among them. In general, such overestimation can be caused either by an underestimation of the white point or by an overestimation of the dark point (see Equation 3). However, this is not a general trend. In fact, a higher reflectance with the lidar can be noticed just for some colors, that do not have any specific common characteristic. This is the reason why the hypothesis that such spectral behavior is related to problems with white and dark points has to be discarded. In particular, the lidar seems to overestimate the reflectance for colors
5, 6, 11, 12, 17 and 18. All these colors belong to the upper-right sector of the color-checker: so, the effect is likely linked to the geometry of the illumination pattern. Although care has been taken to set the R7s lamps in a precise $45^{\circ}: 0^{\circ}$ setup, the geometry of the illumination pattern may alter reflectance estimation, especially if the white spectrum calibration is collected in a different part of the image. In fact, illumination might be not completely uniform both for asymmetries in the direct component (slight deviations from $45^{\circ}: 0^{\circ}$ geometry), and for reflections from walls or furniture in the room. In fact, the lidar is designed to operate in non-controlled environments, so, for example, light reflected from walls or entering from windows in a museum could interfere with light sources. However, the evaluation of these effects is part of the present work and taking them into account is important. The black and white targets are located on the side of the color-checker, so non-uniformity of illumination may affect the white point and black point normalizations. Moreover, the lamps are not exactly point-like, so the light hits the target (which is extended, too) in a range of angles around $45^{\circ}$, and a more complex pattern of the diffuse reflection contribute to the signal detected by the system. An overestimation of the reflectance leads to a higher Y component and then a higher $L^{*}$ coordinate, finally affecting the $\Delta E$. Strictly speaking, a theoretical correction could be derived through the bidirectional reflectance distribution function, but if the light sources are small enough and the background illumination can be considered negligible, it is possible to apply the Lambert cosine law to correct the intensity from any point of the target. However, this is beyond the scope of this work and will not be addressed further. 

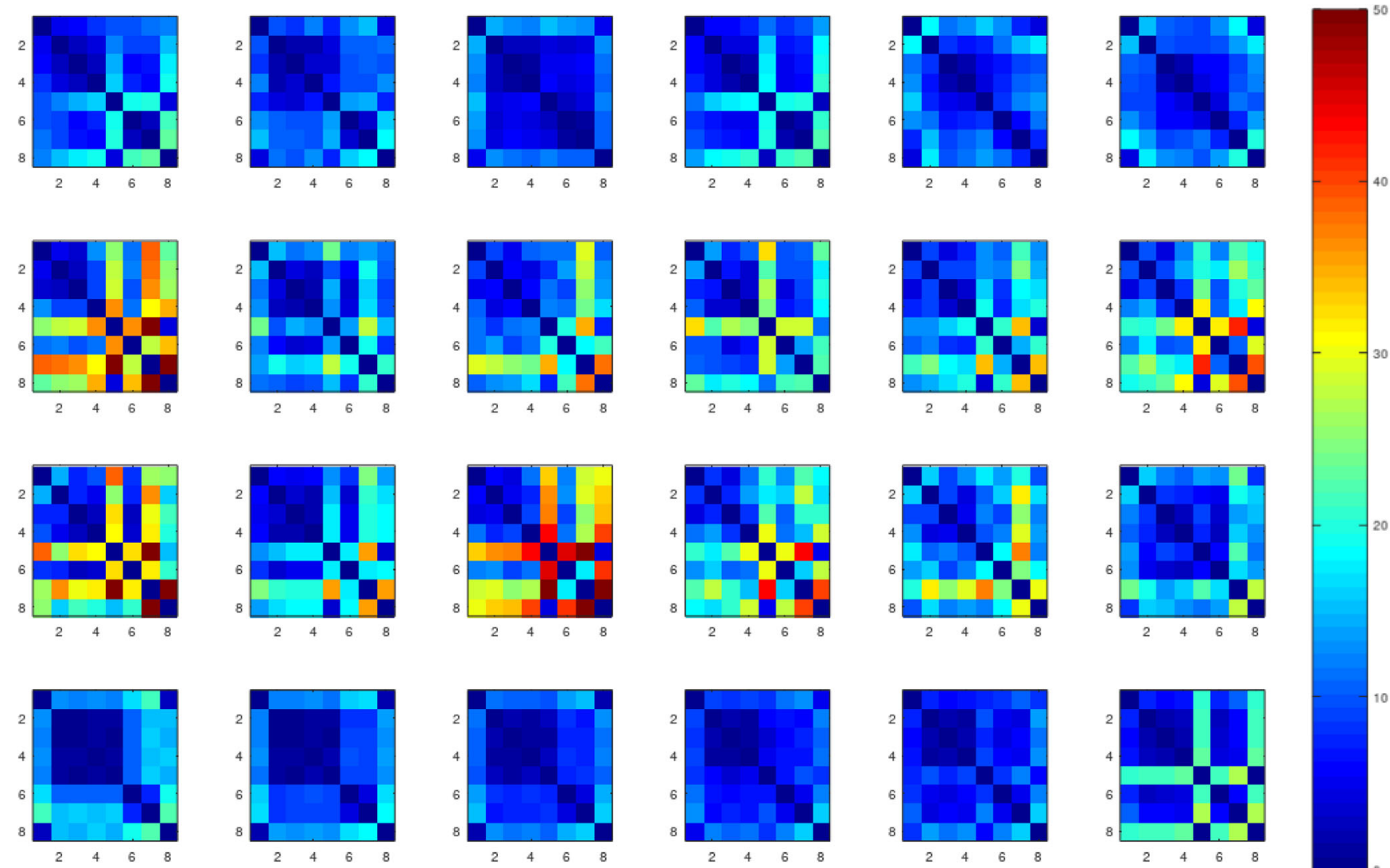

F I G U RE $6 \Delta E$ among different methods for each color. The number of rows and columns represent the measurement method in the following order: 1. Values provided by the color-checker manufacturer; 2. Values calculated from lidar; 3.Values calculated using spectra from ColorTest colorimeter; 4.Values calculated using spectra from fiber optics reflectance spectroscopy system (FORS); 5.Values calculated using spectra from CM-2600d colorimeter; 6. Values directly provided by ColorTest colorimeter; 7. Values directly provided by FORS; 8. Values directly provided by CM-2600d colorimeter

The reflectance spectra obtained for all the target colors listed in Table 1 have been elaborated as explained above and converted to $L^{*} a^{*} b^{*}$ coordinates. After that, the $\Delta E$ among all the datasets can be calculated and compared. Figure 6 summarizes results obtained for all the 24 colors of the color-checker.

In particular, the following $\Delta E$ can be highlighted:

I. $\Delta E$ between $\mathrm{X}$-Rite tabulated data and values obtained with commercial colorimeters with manufacturer software (for every color of the colorchecker).

II. $\Delta E$ between $\mathrm{X}$-Rite tabulated data and values obtained with commercial colorimeters with custom developed software (for every color of the colorchecker).

III. $\Delta E$ between $\mathrm{X}$-Rite tabulated data and values obtained with lidar (for every color of the colorchecker).

From these quantities, the following comparisons will be analyzed:
1. For every color, for every contact instrument, $\Delta E(\mathrm{I})$ vs $\Delta E(\mathrm{II})$

2. For every color, for every contact instrument, $\Delta E(\mathrm{I})$ vs $\Delta E(\mathrm{III})$.

3. For every color, for every contact instrument, $\Delta E$ (II) vs $\Delta E$ (III)

4. For every color, the mean value of $\Delta E$ (I) vs $\Delta E(\mathrm{III})$

5. For every color, the mean value of $\Delta E(\mathrm{II})$ vs $\Delta E(\mathrm{III})$

The results for the first comparison are reported in Figure 7 . The $\Delta E$ values thus obtained show a high variability between them from color to color. For both FORS and ColorTest, the data show, for each color, a higher value for the $\Delta E$ related to the manufacturer algorithm than for the one related to the custom algorithm, while in the case of CM-2600d the $\Delta E$ values related to both the algorithms are similar.

For CM2600d and FORS the manufacturer software uses the spectral ranges 360 to $740 \mathrm{~nm}$ and 380 to $780 \mathrm{~nm}$, respectively, while the custom algorithm processes data in the interval 400 to $700 \mathrm{~nm}$. This is the spectral range provided by the ColorTest, so, for a more 

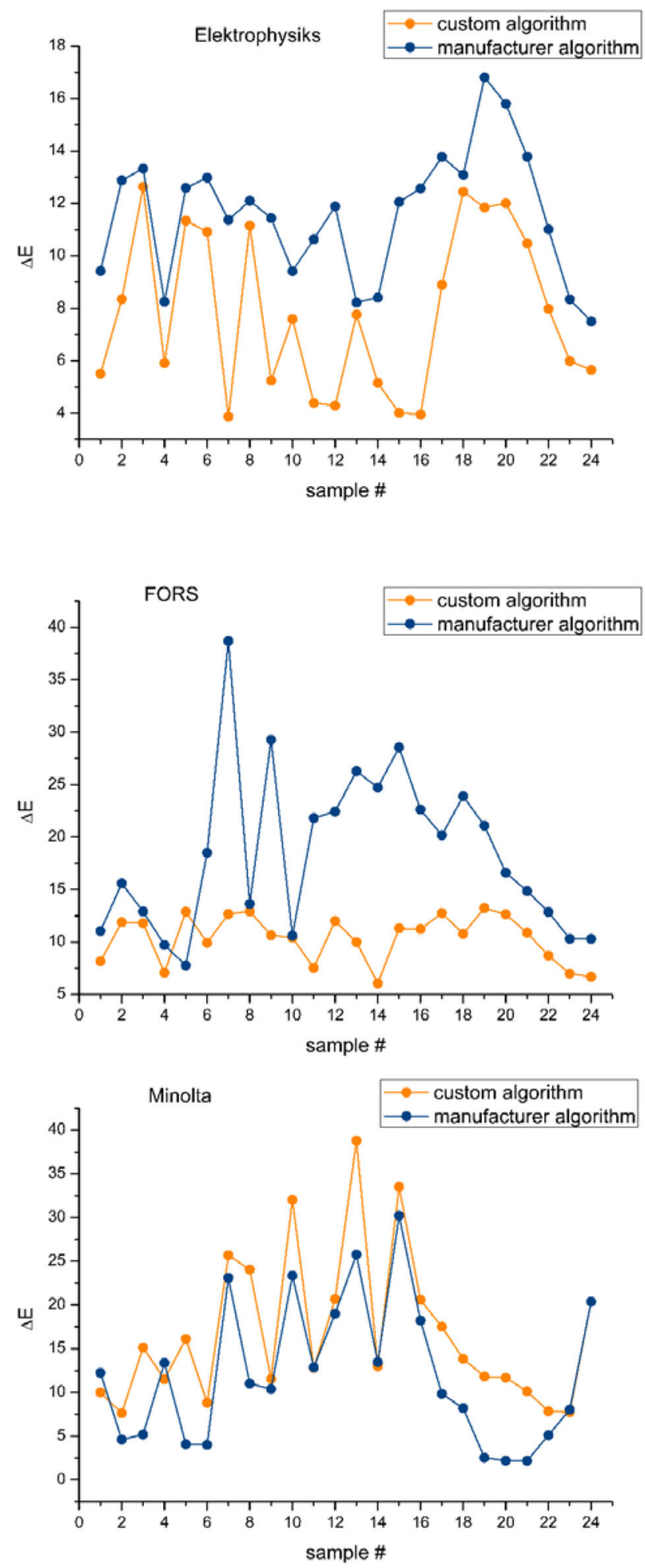

F I G U RE 7 CIELAB color difference $\left(\Delta E^{*}{ }_{\mathrm{ab}}\right)$. In blue the $\Delta E$ between instrumental values and X-Rite tabulated. In orange $\Delta E$ between spectral values elaborated with the developed program and $\mathrm{X}$ Rite tabulated values. For ColorTest (top), for fiber optics reflectance spectroscopy system (FORS) (center) and for CM-2600d (bottom)

significant comparison, the same interval has been chosen also for the other instruments. On the other hand, a sensitivity study performed on selected spectra obtained

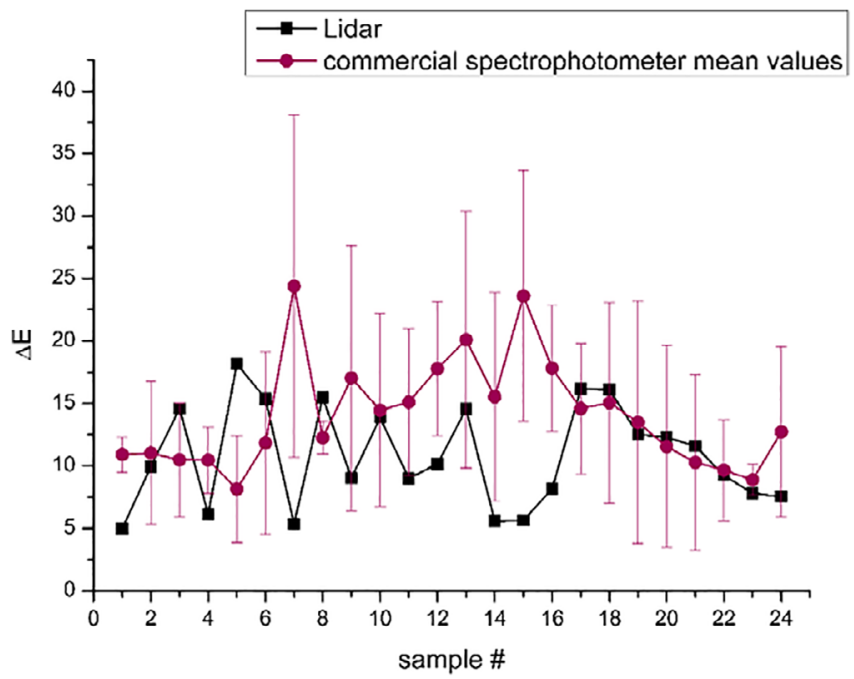

F I G U RE 8 CIELAB color difference $(\Delta E)$ obtained from the comparison with X-Rite tabulated values. Purple curve $\Delta E$ with SD for the mean of commercial measurement systems and black curve $\Delta E$ for the lidar instrument system

with FORS and CM2600d showed that, including the intervals before $400 \mathrm{~nm}$ and after $700 \mathrm{~nm}$ in the custom algorithm, XYZ values undergo a variation of less than $3 \%$, so no appreciable changes in the results on $L^{*} a^{*} b^{*}$ were expected. However, we can see that, while a large difference for the FORS instrument can be noticed, the two sets of data of the CM-2600d are in a good agreement.

In general, the $\Delta E$ values obtained comparing X-Rite tabulated data and experimental ones are much higher than expected for all the systems tested, even though there is not much literature on the subject.

By comparing data in Figure 7, it is possible to notice some trends. For example, colors 7 (orange) and 15 (red) have shown a high color difference with any instrument. Such two values get reduced when calculated with the custom algorithm for ColorTest and FORS.

In Figure 8 the $\Delta E$ values obtained for the three standard instruments operating in contact conditions for each color (by the use of their manufacturer software) were averaged and plotted together with the $\Delta E$ obtained for the lidar scanning system (comparison 4). From the values shown in Table 3 it can be seen that the larger $\Delta E$ are more frequent among those measured with contact instruments (calculated with their own manufacturer software). In particular, the percentage of samples with greater $\Delta E$ for contact systems is around $66.67 \%$ compared to $33.33 \%$ for $\Delta E$ obtained for the lidar system. A similar trend is appreciable only for the samples representing the scale of gray tones (samples 19-24). 
T A B L E $3 \Delta E$ between X-Rite tabulated values and experimental values obtained with the four tested systems with their manufacturer software (illuminant D50 and the $2^{\circ}$ observer)

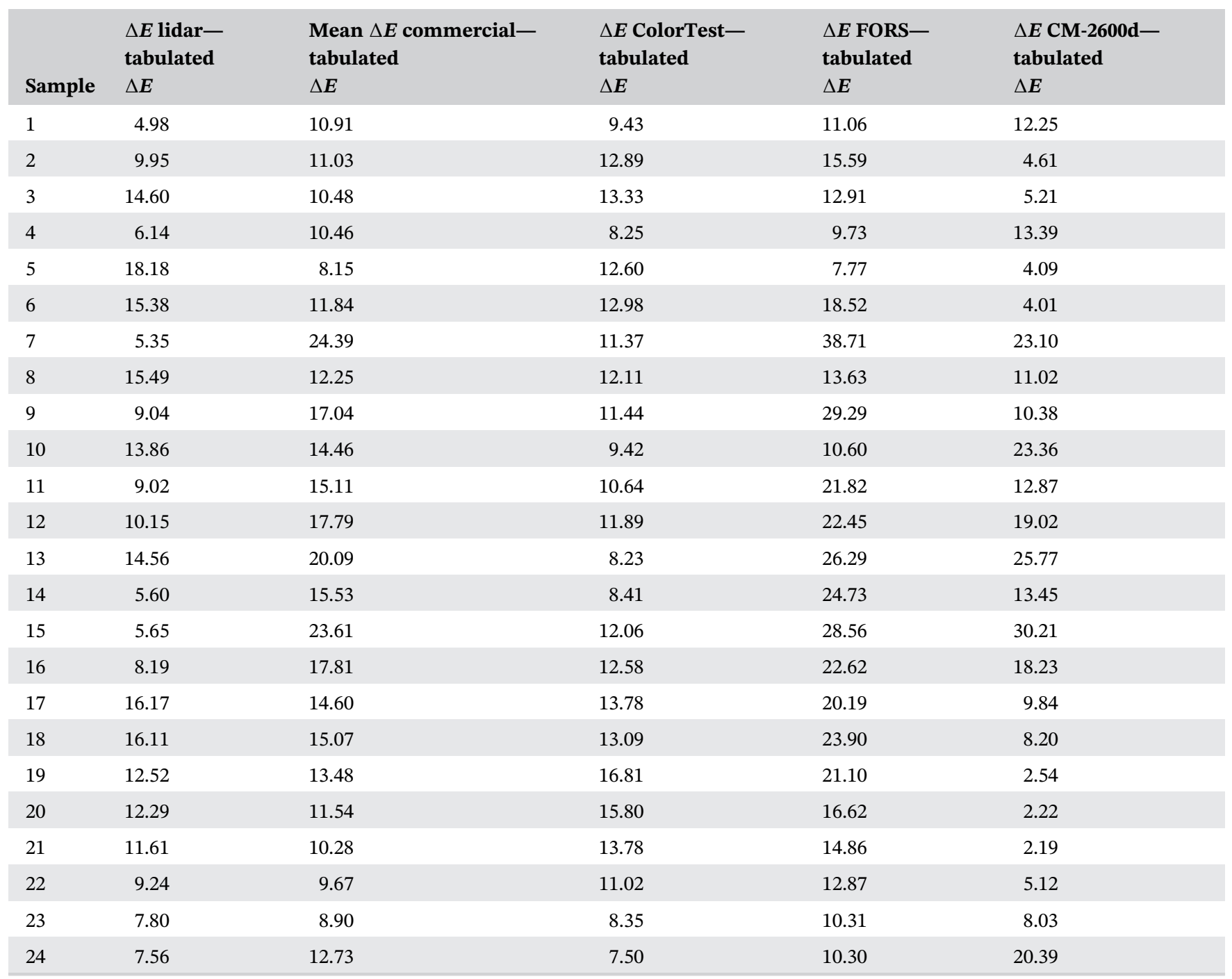

Abbreviation: FORS, fiber optics reflectance spectroscopy system.

The high reproducibility of every single instrument has been already discussed, but Figure 8 underlines how, on the contrary, the SD of the values coming from the average on the data of the three contact instruments is absolutely not negligible: contact tools provide very different results among them, even if they are considered reliable. Such results are reported in Table 3.

Referring to the spectra shown in Figure 5, it is possible to comment the relative data on Table 3. In fact, the two colors represent an interesting example of how, from the trends of the reflectance spectra, it is difficult to predict the results regarding the colorimetric data, and in particular the $\Delta E$ with respect to the X-Rite tabulated values of the color-checker. For color 7 (orange), spectra of the four instruments are very similar, but there is a large variability for the $\Delta E$ values and in particular for
T A B L E 4 Correlation coefficient obtained between $\Delta E$ (X-Rite tabulated data and measured data) calculated for the lidar and $\Delta E$ and for commercial instruments

\begin{tabular}{lllll} 
& ColorTest & CM-2600d & FORS & Lidar \\
\hline ColorTest & 1 & -0.54 & 0.15 & 0.47 \\
CM-2600d & - & 1 & 0.43 & -0.39 \\
\hline FORS & - & - & 1 & -0.23 \\
lidar & - & - & - & 1 \\
\hline
\end{tabular}

Abbreviation: FORS, fiber optics reflectance spectroscopy system.

lidar a much lower value is obtained. Instead, in the case of color 6 (bluish green) the reflectance spectrum of the lidar is far (in terms of intensity, not as trend) from the other ones, but the final $\Delta E$ value is close to those obtained for ColorTest and FORS. 
The Pearson correlation coefficient presented in Table 4 is calculated considering the $\Delta E$ obtained for all the instruments used. The correlation coefficient between the measures is equal to 1 when the data are compared with themselves. These correlation coefficients indicate that the results for all the instruments are different, and at the same time we can see that the results obtainable from commercial instruments do not resemble each other more than they resemble with the data obtained from lidar.

\section{4 | CONCLUSIONS}

The results obtained in this work allowed the estimatation the validity of colorimetric remote measurements carried out with the lidar scanning prototype system developed at the Diagnostics and Metrology Laboratory (FSN-TECFIS-DIM) of ENEA in Frascati. To increase as much as possible the data quality, the casual errors were minimized choosing an area surrounding the point selected from the image of the scanned area; the average and standard deviation were then calculated. Therefore, the accuracy of the lidar measurements has been estimated and it is similar to that of the other instruments used in the study.

The colorimetric measurements obtained with contact instruments usually used for colorimetry are far from showing a perfect agreement among them. However, this result is somehow expected since each instrument performs a slightly different analysis, either because of the geometry of illumination and collection, or because of the retrieval algorithms.

Furthermore, the lidar system works in an even different way, as described, and is designed to operate in uncontrolled environment such as museum halls, catacombs, or even open air. In this work we aimed to understand the capability of this system to obtain reliable colorimetric coordinates of remote samples, in particular when contact measurements are not possible.

In summary, the lidar prototype system has proven to be a valid tool for the colorimetric analysis. More precisely, being a non-invasive tool, it can be very useful especially when standard instruments for colorimetry cannot be used; for example, in cases of highly deteriorated samples, whose manipulation can therefore affect their state of conservation, or when the operator is not allowed to work in contact with the sample (eg, in hostile environments). Moreover, the lidar greatly facilitates the acquisition of data for large surfaces. However, as emerged from this work, great care has to be taken in setting the geometry of the system, since Lambertian law of diffusion may affect the reflectance spectrum of extended targets. For very large targets (frescoes, large paintings, walls), a theoretical correction function could be derived involving the bidirectional reflectance distribution function, possibly in a simplified form.

\section{ACKNOWLEDGMENTS}

The present work has been carried out in the frame of the regional projects COBRA (Latium Region under grant agreement lr13, n.1031) and ADAMO (Research Project on Technologies of Analysis, Diagnostics and Monitoring for the preservation and restoration of Cultural Heritage, in the Centre of Excellence of the District of Technologies for Culture of Latium Region). The authors would like to thank Dr Massimiliano Guarneri for his assistance in colorimetric measurements and for useful discussions.

\section{CONFLICT OF INTEREST}

The author declares that there is no conflict of interest that could be perceived as prejudicing the impartiality of the research reported.

\section{AUTHOR CONTRIBUTIONS}

Valeria Spizzichino and Federico Angelini: Study concept and design. Martina Franchi, Valeria Spizzichino and Anna C. Felici: Measurements. Martina Franchi, Federico Angelini and Valeria Spizzichino: Analysis and interpretation of data. Valeria Spizzichino and Federico Angelini: Drafting of the manuscript. Anna C. Felici and Martina Franchi: Critical revision of the manuscript for important intellectual content.

\section{DATA AVAILABILITY STATEMENT}

Data available on request from the authors

\section{ORCID}

Valeria Spizzichino (D) https://orcid.org/0000-0002-74834716

\section{REFERENCES}

[1] Ohta N, Robertson A. Colorimetry: Fundamentals and Applications. Hoboken, NJ, USA: Wiley; 2006 https://books.google.it/ books?id=U8jeh1uhSHgC.

[2] Johnston-Feller R, Institute GC, Staff GCI. Color Science in the Examination of Museum Objects: Nondestructive Procedures. Los Angeles (USA): Getty Conservation Institute; $2001 \mathrm{https} / /$ books.google.it/books?id=BuMKBpbI77IC.

[3] Coccato A, Moens L, Vandenabeele P. On the stability of mediaeval inorganic pigments: a literature review of the effect of climate, material selection, biological activity, analysis and conservation treatments. Herit Sci. 2017;5(1):1-25. https://doi. org/10.1186/s40494-017-0125-6.

[4] Oleari C. Misurare Il Colore. 2nd ed. Milano(Italy): Hoepli; 2008. 
[5] Internationale de l'éclairage C. Recueil Des Travaux et Compte Rendu Des Séances: 8. Session. Cambridge: University Press; 1932.

[6] CIE Publication S014-2:2006. Colorimetry, Standard Illuminants for Colorimetry. 2nd ed. Vienna, Austria: CIE Central Bureau; 2006.

[7] CIE Publication N.176:2006. Geometrical Tolerances for Colour Measurement. Vienna, Austria: CIE Central Bureau; 2006.

[8] Schanda J. Colorimetry: Understanding the CIE System. Hoboken, NJ (USA): Wiley; 2007 https://books.google.it/books?id= uZadszSGe9MC.

[9] McLaren K. The development of the CIE 1976 (L* a* b*) uniform colour space and colour-difference formula. Color Technol. 1976;92(9):338-341.

[10] X-Rite color-checker, https://www.xrite.com/categories/ calibration-profiling/colorchecker-classic.

[11] Bacci M, Picollo M, Trumpy G, Tsukada M, Kunzelman D. Non-invasive identification of white pigments on 20Thcentury oil paintings by using fiber optic reflectance spectroscopy. J Am Inst Conserv. 2007;46(1):27-37. https://doi.org/10. 1179/019713607806112413.

[12] Maynez-Rojas M, Casanova-González E, Ruvalcaba-Sil J. Identification of natural red and purple dyes on textiles by fiberoptics reflectance spectroscopy. Spectrochim Acta A Mol Biomol Spectrosc. 2017;178:239-250. https://doi.org/10.1016/j.saa. 2017.02.019.

[13] CIE Publication N.015:2018. Colorimetry. 4th ed. Vienna, Austria: CIE Central Bureau; 2018.

[14] Perales E, Chorro E, Viqueira V, Martínez-Verdú F. Reproducibility comparison among multiangle spectrophotometers. Color Res Appl. 2012;38(3):160-167. https://doi.org/10.1002/ col.21719.

[15] Xu H, Zhang H, Rao B, Yang Y, Zhang Z. An automatic white balance algorithm based on pixel luminance and chromaticity. In: Wang Y, Huang Q, Peng Y, eds. Image and Graphics Technologies and Applications. Singapore: Springer Singapore; 2019:353-359p.

\section{AUTHOR BIOGRAPHIES}

Angelini Federico, physicist, $\mathrm{PhD}$, in remote sensing, has been working with Raman, Rayleigh-Mie and fluorescence lidar for atmospheric, marine and security applications since 2001 .

Anna C. Felici, with a degree in physics, is a researcher in the fields of solid-state physics and archaeometry. She is Director of the Laboratory for Non Destructive Analyses and Archaeometry - LANDA "Sebastiano Sciuti" of the University of Rome "La Sapienza."

Martina Franchi graduated with a major in Science and Technology for the Conservation of Cultural Heritage from "La Sapienza" University, Rome, Italy, in 2019.

Valeria Spizzichino, $\mathrm{PhD}$, in chemical sciences, has been working since 2002 on laser spectroscopy, both in design and development of stand-off and shortrange systems, in different field of applications, as Cultural Heritage, environment, security, forensics.

How to cite this article: Angelini F, Felici AC, Franchi M, Spizzichino V. Remote colorimetric measurements by hyperspectral lidar compared to contact conventional colorimetry. Color Res Appl. 2020;1-13. https://doi.org/10.1002/col.22600 